\title{
REFLECTED BACKWARD STOCHASTIC DIFFERENTIAL EQUATIONS WITH RESISTANCE
}

\author{
By ZhONGMIN QIAN ${ }^{1}$ AND MingYu XU ${ }^{2}$ \\ University of Oxford and Chinese Academy of Science
}

\begin{abstract}
In this article, we study a class of reflected backward stochastic differential equations (introduced in El Karoui et al. [Ann. Probab. 25 (1997) 702-737], RBSDE for short) with nonlinear resistance by means of Skorohod's equation. The advantage of this approach lies in its pathwise nature and, therefore, provides additional information about solutions of RBSDE. As an application of our approach, we will consider reflected backward problems with resistance as well. This class of RBSDEs possess significance in the super-hedging with wealth constraint.
\end{abstract}

1. Introduction. The study of backward stochastic differential equations (BSDEs) was initiated in Bismut [2] where a linear version of BSDEs is formulated in order to address the stochastic maximal principles. Pardoux and Peng proved the existence and uniqueness of adapted solutions to BSDEs with drivers which are Lipschitz continuous. After that, the theory of BSDEs associated with semilinear parabolic equations however was established in Peng [17] and Pardoux and Peng [15]. El Karoui, Kapoudjian, Pardoux, Peng and Quenez [3] have introduced the following class of reflected BSDEs with continuous barriers:

$$
\left\{\begin{array}{l}
Y_{t}=\xi+\int_{t}^{T} f\left(s, Y_{s}, Z_{s}\right) d s+K_{T}-K_{t}-\int_{t}^{T} Z_{s} d B_{s}, \\
Y_{t} \geq S_{t} \quad \forall t \in[0, T],
\end{array}\right.
$$

where $f:[0, T] \times \Omega \times \mathbb{R} \times \mathbb{R}^{d} \rightarrow \mathbb{R}$ called the driver of $(1.1), B=\left(B^{1}, \ldots, B^{d}\right)$ is a $d$-dimensional standard Brownian motion on a complete probability space $(\Omega, \mathcal{F}, \mathbb{P})$, and $\left(\mathcal{F}_{t}\right)_{t \geq 0}$ is the Brownian filtration associated with $B . \xi$ which is $\mathcal{F}_{T}$-measurable, is the terminal value of the problem, and $S$, a given continuous process, serves as the reflecting boundary.

A solution to (1.1) is a triple $(Y, Z, K)$ of adapted stochastic processes in $\mathbb{R}^{1+d+1}$, which satisfies the stochastic integral equation (1.1), where $K$ is nondecreasing and continuous, with initial zero.

Received March 2014; revised April 2017

${ }^{1}$ Research supported in part by EPSRC grant EP/F029578/1 and an ERC grant Esig ID 291244.

${ }^{2}$ Supported by NSFC Grant $11371350 / \mathrm{A} 0110$ and in part by a LMS scheme 2 grant. MSC2010 subject classifications. Primary 60H10, 60J45.

Key words and phrases. Brownian motion, local time, optional dual projection, reflected BSDE, Skorohod's equation. 
The role of $K$ is to push the process $Y$ upward with minimal cost, and to keep it stay above $S$ in such way that

$$
\int_{0}^{T}\left(Y_{s}-S_{s}\right) d K_{s}=0
$$

In [3], a penalization procedure is applied to construct approximations of a solution to (1.1), and the Picard-type iteration is used to solve an optimal stopping problem at each step to ensure the constraint that $Y \geq S$ is satisfied. There are many papers published over the past years in which similar reflected BSDEs have been considered under weaker assumptions, based on the penalization technique. In [14], Matoussi has studied the case in which the driver $f$ is of linear growth, but not necessary Lipschitz continuous, while in [10], Lepeltier, Matoussi and the second author of the present paper have considered the case where $f(t, y, z)$ is Lipschitz in $z$ and monotone in $y$. In particular, $f$ may be neither Lipschitz continuous nor of linear growth in $y$. RBSDE with a driver $f(t, y, z)$ which is of quadratic growth in $z$ has been considered in $[9,12]$ and [18]. In another direction, different barrier conditions have been considered, for example, in [6] and [11], the case of discontinuous barrier $S$ is considered, and in [16] even more general barriers have been studied.

In Bank and El Karoui [1], a new type of reflected BSDEs has been studied by means of Skorohod's obstacle problem, which was studied further by Ma and Wang [13] in a more general setting. Ma and Wang formulated the following problem:

$$
\left\{\begin{array}{l}
Y_{t}=X_{T}+\int_{t}^{T} f\left(s, Y_{s}, Z_{s}, A_{s}\right) d s-\int_{t}^{T} Z_{s} d B_{s}, \\
Y_{t} \leq X_{t} \quad \forall t \in[0, T],
\end{array}\right.
$$

where $A$ is an increasing process starting from $-\infty$, which satisfies that $\int_{t}^{T}\left(X_{S}-\right.$ $\left.Y_{s}\right) d A_{s}=0$. $A$ drives $Y$ through the generator $f$ (which is decreasing in $A$ ) downwards to ensure that $Y \leq X$. Therefore, $A$ is considered as the "density" of the reflecting force. In [13], a solution in a small-time duration has been obtained and the uniqueness has been established as well.

If $(Y, Z, K)$ is a solution of (1.1), according to Skorohod's equation, $K$ has an explicit representation in terms of $Y$ and $Z$ given by

$$
\begin{aligned}
K_{t}= & \max \left[0, \max _{0 \leq s \leq T}\left\{-\left(\xi+\int_{s}^{T} f\left(r, Y_{r}, Z_{r}\right) d r-S_{s}-\int_{s}^{T} Z_{r} d B_{r}\right)\right\}\right] \\
& -\max \left[0, \max _{t \leq s \leq T}\left\{-\left(\xi+\int_{s}^{T} f\left(r, Y_{r}, Z_{r}\right) d r-S_{s}-\int_{s}^{T} Z_{r} d B_{r}\right)\right\}\right],
\end{aligned}
$$

which shows how the force $K$ pushes the solution $Y$ with respect to the barrier $S$; see Section 2 below. 
Thanks to the theory of optional dual projections (for details about the general theory of stochastic processes, see, e.g., [7]), Skorohod's equation (1.4) can be applied to the construction of a Picard iteration which solves the reflected BSDE (1.1). This approach allows us to include the force $K$ into the driver which is an analogous of (1.3). Explicitly, we study the following type of reflected BSDE:

$$
Y_{t}=\xi+\int_{t}^{T} f\left(s, Y_{s}, Z_{s}, H(K)_{s}\right) d s+K_{T}-K_{t}-\int_{t}^{T} Z_{s} d B_{s}, \quad t \leq T
$$

subject to the constraint that

$$
Y_{t} \geq S_{t} \quad \text { for } t \leq T \quad \text { and } \quad \int_{0}^{T}\left(Y_{t}-S_{t}\right) d K_{t}=0
$$

Here, the force is still applied via an increasing process $K$, where $K$ increases only on the duration that $Y$ hits the barrier $S$, and $K$ appears in the driver $f$ through a linear mapping $H$ as well. $f$ has instant effect from the process $H(K)$, via $H$ which may report some long term influence of $K$. If $f \circ H$ is decreasing in $K$, then there will be an extra force applying on $Y$, otherwise, if $f \circ H$ is increasing in $K$, then there is a reversed force coming from $K$ which resists the linear force $K$. In general, the reflected BSDE (1.5) is considered as an equation with resistance. Since $Y \geq S$ has to be satisfied, the extra force induced by $H(K)$ has to be controlled, characterized by the magnitude of Lipschitz constant in $K$, which thus cannot be arbitrary.

The paper is organized as follows. We recall in Section 2 Tanaka's formula and Skorohod's equation to give various formulae for $K$. In Section 3, we introduce a type of reflected BSDEs with resistance and prove the existence and uniqueness of the solution. In Section 4, we give some application of reflected BSDE in finance, and in Section 5 a very interesting case of reflected BSDE is introduced and studied.

2. Local and reflected local times. Let $T>0$ be a terminal time, and $\mathcal{P}$ be the $\sigma$-algebra of predictable subsets of $[0, T] \times \Omega$ with respect to the filtration $\left(\mathcal{F}_{t}\right)_{t \geq 0}$. We introduce first the following spaces of random processes over $\left(\Omega, \mathcal{F}, \mathcal{F}_{t}, \mathbb{P}\right) . \mathcal{L}^{2}\left(\mathcal{F}_{t}\right)$ denotes the space of all $\mathcal{F}_{t}$-measurable, square integrable real random variables, $\mathcal{M}^{2}$ the space of (continuous) square integrable martingales (up to time $T$ ), and $\mathcal{H}_{d}^{2}(0, T)$ the space of $\mathbb{R}^{d}$-valued predictable processes $\psi$ such that $\mathbb{E} \int_{0}^{T}|\psi(t)|^{2} d t<\infty . \mathcal{S}^{2}(0, T)$ denotes the space of all continuous semimartingales (with running time $[0, T]$ ) over $\left(\Omega, \mathcal{F}, \mathcal{F}_{t}, \mathbb{P}\right)$, and $\mathcal{A}^{2}(0, T)$ the space of all $\mathcal{F}_{T}$-measurable continuous and increasing processes $K$ with initial zero such that $\mathbb{E} K_{T}^{2}<\infty$. Finally, $\mathcal{A}_{\mathcal{F}}^{2}(0, T)$ denotes the space of $\mathcal{F}_{t}$-progressively measurable processes in $\mathcal{A}^{2}(0, T)$.

The reflected backward stochastic differential equation (RBSDE or reflected BSDE in short) considered in El Karoui et al. [3] is a stochastic integral equation:

$$
Y_{t}=\xi+\int_{t}^{T} f\left(s, Y_{s}, Z_{s}\right) d s+K_{T}-K_{t}-\int_{t}^{T} Z_{s} d B_{S}
$$


for $t \leq T$, subject to the constraint that

$$
Y_{t} \geq S_{t} \quad \text { for } t \leq T \quad \text { and } \quad \int_{0}^{T}\left(Y_{t}-S_{t}\right) d K_{t}=0,
$$

where $S$ is a continuous semimartingale such that $\sup _{t \leq T} S_{t}^{+}$is square integrable, and the terminal data $\xi \in \mathcal{L}^{2}\left(\mathcal{F}_{T}\right)$. The driver $f(t, y, z)$ is global Lipschitz in $(y, z)$ uniformly in $t \in[0, T]$ and $\omega \in \Omega$.

By a solution $(Y, Z, K)$ of the terminal problem (2.1)-(2.2), we mean that $Y \in \mathcal{S}^{2}(0, T), K \in \mathcal{A}_{\mathcal{F}}^{2}(0, T), K$ is optional, and $Z \in \mathcal{H}_{d}^{2}(0, T)$ which satisfy the stochastic integral equation (2.1) with time $t$ running from 0 to $T$, and the constraint (2.2).

The constraint (2.2) implies that $\xi-S_{T}$ must be nonnegative, and the second condition in (2.2) says that $K$ has no charge on $\left\{t \in[0, T]: Y_{t}>S_{t}\right\}$ and increases only on $\left\{t: Y_{t}=S_{t}\right\}$, which is equivalent to say that $\int_{0}^{t} 1_{\left\{Y_{s}-S_{s}=0\right\}} d K_{s}=K_{t}$ for $0 \leq t \leq T$.

2.1. Tanaka's formula. If $X$ is a continuous semimartingale, then $L^{X}$ denotes the local time of the continuous semimartingale $X-S$ at zero, which may be defined via Tanaka's formula:

$$
\left|X_{t}-S_{t}\right|=\left|X_{0}-S_{0}\right|+\int_{0}^{t} \operatorname{sgn}\left(X_{s}-S_{s}\right) d\left(X_{s}-S_{s}\right)+2 L_{t}^{X},
$$

where $\operatorname{sgn}(r)=-1$ for $r \leq 0$ and $\operatorname{sgn}(r)=1$ for $r>0$. Then

$$
\left(X_{t}-S_{t}\right)^{-}=\left(X_{0}-S_{0}\right)^{-}-\int_{0}^{t} 1_{\left\{X_{s} \leq S_{s}\right\}} d\left(X_{s}-S_{s}\right)+L_{t}^{X} .
$$

The following results have already appeared in [3], in a slightly different form.

Proposition 2.1. Suppose that $Y_{t}=\int_{0}^{t} Z_{s} d B_{s}+V_{t}$ and $S_{t}=\int_{0}^{t} \sigma_{s} d B_{s}+A_{t}$ are two continuous semimartingales, where $V$ and $A$ are continuous, adapted with finite variations. Suppose that $Y \geq S$. Then

$$
L_{t}^{Y}=\int_{0}^{t} 1_{\left\{Y_{s}=S_{s}\right\}} d\left(V_{s}-A_{s}\right)
$$

and

$$
1_{\left\{Y_{t}=S_{t}\right\}}\left(Z_{t}-\sigma_{t}\right)=0 \text {. }
$$

Proof. Since $Y-S \geq 0,(Y-S)^{-}=0$. Applying Tanaka's formula (2.4) to $Y$, we obtain

$$
\begin{aligned}
L_{t}^{Y} & =\int_{0}^{t} 1_{\left\{Y_{s}=S_{s}\right\}} d\left(Y_{s}-S_{S}\right) \\
& =\int_{0}^{t} 1_{\left\{Y_{s}=S_{s}\right\}} d\left(V_{s}-A_{s}\right)+\int_{0}^{t} 1_{\left\{Y_{s}=S_{s}\right\}}\left(Z_{s}-\sigma_{s}\right) d B_{s} .
\end{aligned}
$$


Since $L$ is increasing, the martingale part must be zero, and (2.6) holds. Therefore, (2.5) follows as well.

COROLlary 2.2. Suppose that $Y$ and $S$ are two semimartingales:

$$
Y_{t}=Y_{0}-\int_{0}^{t} f_{s} d s-K_{t}+\int_{0}^{t} Z_{s} d B_{s}
$$

and $S=N+A$ ( $N$ is the martingale part of $S$ and $A$ is its finite variation part), where $\left(f_{t}\right)_{t \in[0, T]}$ is optional, $\mathbb{E}\left(\int_{0}^{T} f_{s}^{2} d s\right)<\infty, Z \in \mathcal{H}^{d}(0, T), Y_{0} \in \mathcal{L}^{2}\left(\mathcal{F}_{0}\right), K \in$ $\mathcal{A}_{\mathcal{F}}^{2}(0, T)$ is adapted and $\int_{0}^{t} 1_{\left\{Y_{s}=S_{s}\right\}} d K_{s}=K_{t}$. Suppose that $Y \geq S$. Then

$$
K_{t}=-\int_{0}^{t} 1_{\left\{Y_{s}=S_{s}\right\}} f_{s} d s-\int_{0}^{t} 1_{\left\{Y_{s}=S_{s}\right\}} d A_{s}-L_{t}^{Y}
$$

and

$$
K_{t}=-\int_{0}^{t} 1_{\left\{Y_{s}=S_{S}\right\}} f_{s} d s-\int_{0}^{t} 1_{\left\{Y_{s}=S_{s}\right\}} d Y_{s}+\int_{0}^{t} 1_{\left\{Y_{s}=S_{s}\right\}} d N_{s}
$$

If in addition, $A_{t}=\int_{0}^{t} u_{s} d s$, for some $u \in \mathcal{H}_{d}^{2}(0, T)$, then $K$ and $L^{Y}$ are absolute continuous with respect to the Lebesgue measure. Hence there exists a progressive measurable process $\alpha$ valued in $[0,1]$, such that

$$
0 \leq k_{s}=\alpha_{s} 1_{\left\{Y_{s}=S_{s}\right\}}\left(f_{s}+u_{s}\right)^{-}
$$

for $s \in[0, T]$, where $k$ is the density process of $K$, that is, $K_{t}=\int_{0}^{t} k_{s} d s$.

2.2. Skorohod's equation. The most useful form for $K$ in our study is however the representation formula given by Skorohod's equation (see equation (1) in [3]).

Suppose that $Y$ and $S$ are two continuous semimartingales, satisfying $Y \geq S$, and suppose that $Y$ is given by (2.7).

Let $y_{t}=Y_{T-t}-S_{T-t}, L_{t}=K_{T}-K_{T-t}$ and

$$
x_{t}=\int_{T-t}^{T} f_{s} d s-\int_{T-t}^{T} Z_{s} d B_{s}+S_{T}-S_{T-t} .
$$

Then $L_{0}=0, t \rightarrow L_{t}$ increases only on $\left\{t: y_{t}=0\right\}, y_{t} \geq 0, \eta=Y_{T}-S_{T} \geq 0$, $x_{0}=0$, and

$$
y_{t}=\eta+x_{t}+L_{t} .
$$

According to Skorohod's equation (Lemma 6.14, page 210 in [8], with the convention that $x_{t}=x_{T}, y_{t}=y_{T}$ and $L_{t}=L_{T}$ for $t \geq T$ )

$$
L_{t}=\max \left[0, \max _{0 \leq s \leq t}\left\{-\left(\eta+x_{s}\right)\right\}\right] \quad \forall t \geq 0 \text {. }
$$

That is,

$$
L_{t}=\max \left[0, \max _{T-t \leq s \leq T}\left\{-\left(Y_{T}+\int_{s}^{T} f_{r} d r-S_{s}-\int_{s}^{T} Z_{r} d B_{r}\right)\right\}\right]
$$


for $0 \leq t \leq T$. We may recover $K_{t}=L_{T}-L_{T-t}$ and in fact

$$
\begin{aligned}
K_{t}= & \max \left[0, \max _{0 \leq s \leq T}\left\{-\left(Y_{T}+\int_{s}^{T} f_{r} d r-S_{s}-\int_{s}^{T} Z_{r} d B_{r}\right)\right\}\right] \\
& -\max \left[0, \max _{t \leq s \leq T}\left\{-\left(Y_{T}+\int_{s}^{T} f_{r} d r-S_{s}-\int_{s}^{T} Z_{r} d B_{r}\right)\right\}\right] .
\end{aligned}
$$

By using (2.14) and the theory of optional dual projections, we may construct a Picard iteration for the problem $(1.5,1.6)$, which will be developed in the following sections.

3. Reflected BSDE with nonlinear resistance. Let us consider the reflected BSDE with resistance:

$$
Y_{t}=\xi+\int_{t}^{T} f\left(s, Y_{s}, Z_{s}, H(K)_{s}\right) d s+K_{T}-K_{t}-\int_{t}^{T} Z_{s} d B_{s}
$$

for $t \leq T$, subject to the constraint that

$$
Y_{t} \geq S_{t} \quad \text { for } t \leq T \quad \text { and } \quad \int_{0}^{T}\left(Y_{t}-S_{t}\right) d K_{t}=0
$$

where the given process $S$ is a continuous semimartingale such that $\sup _{t \leq T} S_{t}^{+}$is square integrable, and $\xi \in \mathcal{L}^{2}\left(\mathcal{F}_{T}\right)$.

Assume that $f$ is globally Lipschitz continuous,

$$
\left|f(s, y, z, h)-f\left(s, y^{\prime}, z^{\prime}, h^{\prime}\right)\right| \leq C_{1}\left(\left|y-y^{\prime}\right|+\left|z-z^{\prime}\right|\right)+C_{2}\left|h-h^{\prime}\right|
$$

for all $y, y^{\prime}, z, z^{\prime}, h, h^{\prime}$, where $C_{1}$ and $C_{2}$ are two constants, and assume that $\mathbb{E} \int_{0}^{T} f_{0}(t)^{2} d t<\infty$, where

$$
f_{0}(t) \equiv f(t, 0,0,0)
$$

$H$ is a mapping defined on $\mathcal{A}^{2}(0, T)$, which is $L^{\infty}$-Lipschitz (this sort of Lipschitz conditions is often used in numerical solutions of BSDE,; see, e.g., [19]): there exists a constant $C_{3}$, such that

$$
\left|H(a)_{s}-H\left(a^{\prime}\right)_{s}\right| \leq C_{3} \sup _{0 \leq t \leq T}\left|a_{t}-a_{t}^{\prime}\right|
$$

for any continuous increasing functions $a$. and $a_{\text {. }}^{\prime}$. Suppose that $H(a)$. is $\mathcal{F}_{t^{-}}$progressively measurable with $\mathbb{E}\left[\sup _{0 \leq t \leq T}\left|H(a)_{t}\right|\right]<\infty$. Then $H(K)$ has the same measurability as $K$ with respect to the filtration $\left(\mathcal{F}_{t}\right)_{0 \leq t \leq T}$. Furthermore, if $K \in \mathcal{A}_{\mathcal{F}}^{2}(0, T)$, then $H(K) \in S^{2}(0, T)$. Here are two simple but important examples: 
(i) $H(K)_{s}=K_{s}$, with $C_{3}=1$.

(ii) $H(K)_{s}=K_{s \wedge \tau}$, with $C_{3}=1$, where $\tau$ is a stopping time values in $[0, T]$.

By a solution triple $(Y, Z, K)$ of the terminal problem (3.1), we mean that $Y \in$ $\mathcal{S}^{2}(0, T), K \in \mathcal{A}_{\mathcal{F}}^{2}(0, T), K$ is optional, $Z \in \mathcal{H}_{d}^{2}(0, T)$, and $(Y, Z, K)$ satisfies the stochastic integral equations (3.1) with time $t$ running from 0 to $T$.

An additional feature of the reflected BSDE (2.1) is the dependence of the driver with respect to the local time $K$. The integral equation (3.1) is not local in time, since $K$ will be path dependent over the whole range $[0, T]$. This is the reason why we have to require the Lipschitz constant $C_{2}$ in (3.3) to be small in order to ensure the existence of a solution.

According to (2.14), if ( $Y, Z, K)$ is a solution of the problem (3.1)-(3.2), then we must have

$$
\begin{aligned}
K_{t}= & \max \left[0, \max _{0 \leq s \leq T}\left\{-\left(\xi-S_{s}\right.\right.\right. \\
& \left.\left.\left.+\int_{s}^{T} f\left(r, Y_{r}, Z_{r}, H(K)_{r}\right) d r-\int_{s}^{T} Z_{r} d B_{r}\right)\right\}\right] \\
& -\max \left[0, \max _{t \leq s \leq T}\left\{-\left(\xi-S_{s}\right.\right.\right. \\
& \left.\left.\left.+\int_{s}^{T} f\left(r, Y_{r}, Z_{r}, H(K)_{r}\right) d r-\int_{s}^{T} Z_{r} d B_{r}\right)\right\}\right] .
\end{aligned}
$$

3.1. Picard's iteration via Skorohod's equation. We show the existence of a solution by constructing an appropriate (non)linear mapping defined by the stochastic integral equation (3.1), so that a solution is given as its fixed point. The iteration procedure we present through Skorohod's equation is equivalent to solve a reflected BSDE whose coefficient is a given process. However, the iteration procedure through Skorohod's equation gives us a better way to understand how to find the adapted solution of reflected BSDE from nonadapted processes, which is entirely based on their trajectories.

Suppose that $Y \in \mathcal{S}^{2}(0, T), Z \in \mathcal{H}_{d}^{2}(0, T), K \in \mathcal{A}^{2}(0, T)$, and suppose that $Y \geq S$. After iteration once we will obtain

$$
(\tilde{Y}, \tilde{Z}, \tilde{K}) \in \mathcal{S}^{2}(0, T) \times \mathcal{H}_{d}^{2}(0, T) \times \mathcal{A}^{2}(0, T)
$$

and $\int_{0}^{\cdot} \tilde{Z} d B$ is the martingale part of $\tilde{Y}$. Thus we may assume, without losing generality, that $M_{t}-M_{0}=\int_{0}^{t} Z_{s} d B_{s}$ is the martingale part of $Y$, although we will 
consider $Y, Z, K$ as independent elements. According to (3.6), define

$$
\begin{aligned}
\hat{K}_{t}= & \max \left[0, \max _{0 \leq s \leq T}\left\{-\left(\xi+\int_{s}^{T} f\left(r, Y_{r}, Z_{r}, H(K)_{r}^{b}\right) d r\right.\right.\right. \\
& \left.\left.\left.-S_{s}-\int_{s}^{T} Z_{r} d B_{r}\right)\right\}\right] \\
& -\max \left[0, \max _{t \leq s \leq T}\left\{-\left(\xi+\int_{s}^{T} f\left(r, Y_{r}, Z_{r}, H(K)_{r}^{b}\right) d r\right.\right.\right. \\
& \left.\left.\left.-S_{s}-\int_{s}^{T} Z_{r} d B_{r}\right)\right\}\right],
\end{aligned}
$$

where the optional projection $H(K)^{\text {b }}$. of $H(K)$., which is a right-continuous modification of $t \rightarrow \mathbb{E}\left(H(K)_{t} \mid \mathcal{F}_{t}\right)$, is used in place of $H(K)$, as we do not assume that $K$ is optional, but we want to ensure that the arguments of $f$ are optional. This definition is crucial to make our approach without penalization work; see below the proof of Proposition 3.5.

Next, we define $\tilde{M}$ and $\tilde{Y}$. The natural way to define $\tilde{Y}$ is to use the right-hand side of (3.1), that is,

$$
\hat{Y}_{t}=\xi+\int_{t}^{T} f\left(s, Y_{s}, Z_{s}, H(K)_{s}^{b}\right) d s+\hat{K}_{T}-\hat{K}_{t}-\int_{t}^{T} Z_{s} d B_{s} .
$$

$\hat{Y}$ is however not necessarily adapted. Therefore, we define $\tilde{Y}$ to be its optional projection $\hat{Y}^{b}$, that is,

$$
\begin{aligned}
\tilde{Y}_{t} & =\mathbb{E}\left\{\xi+\int_{t}^{T} f\left(s, Y_{s}, Z_{s}, H(K)_{s}^{\mathrm{b}}\right) d s+\hat{K}_{T}-\hat{K}_{t}-\int_{t}^{T} Z_{s} d B_{s} \mid \mathcal{F}_{t}\right\} \\
& =\mathbb{E}\left\{\xi+\int_{t}^{T} f\left(s, Y_{s}, Z_{s}, H(K)_{s}^{\mathrm{b}}\right) d s+\hat{K}_{T}-\hat{K}_{t} \mid \mathcal{F}_{t}\right\} .
\end{aligned}
$$

According to Skorohod's equation, $\hat{Y} \geq S$, so is $\tilde{Y}$. Therefore, the mapping $Y \rightarrow \tilde{Y}$ preserves the constraint that $\tilde{Y} \geq S . \hat{K}$ increases only on $\left\{t: \hat{Y}_{t}-S_{t}=0\right\}$, which however does not necessarily coincide with the level set $\left\{t: \tilde{Y}_{t}-S_{t}=0\right\}$.

By our assumptions, $\hat{K}$ is $\mathcal{F}_{T}$-measurable, continuous and increasing, and its optional projection $\hat{K}^{b}$ and its dual optional projection $\hat{K}^{o}$ exist. The dual optional projection $\hat{K}^{o}$ is continuous and increasing with initial zero, while the optional projection $\hat{K}^{b}$ is right continuous but not necessarily increasing. Their difference $\tilde{N}=\hat{K}^{b}-\hat{K}^{o}$ is a martingale which must be continuous. Hence the optional pro- 
jection $\hat{K}^{b}$ is continuous as well. Moreover the mapping which sends $\hat{K}$ to $\hat{K}^{b}$ is a contraction with respect to the $L^{p}$-norm for every $p \geq 1$.

By (3.9), the semimartingale decomposition of $\tilde{Y}$ is given by

$$
\begin{aligned}
\tilde{Y}_{t}= & \mathbb{E}\left\{\xi+\tilde{K}_{T}+\int_{0}^{T} f\left(s, Y_{s}, Z_{s}, H(K)_{s}^{b}\right) d s \mid \mathcal{F}_{t}\right\}-\tilde{N}_{t} \\
& -\hat{K}_{t}^{o}-\int_{0}^{t} f\left(s, Y_{s}, Z_{s}, H(K)_{s}^{b}\right) d s .
\end{aligned}
$$

Let $\tilde{K}_{t}=\hat{K}_{t}^{o}$. Then the martingale part of $\tilde{Y}$ is given by

$$
\tilde{M}_{t}=\mathbb{E}\left\{\xi+\tilde{K}_{T}+\int_{0}^{T} f\left(s, Y_{s}, Z_{s}, H(K)_{s}^{b}\right) d s \mid \mathcal{F}_{t}\right\}-\tilde{N}_{t}
$$

which in turn defines the density predictable process $\tilde{Z}$ by Itô's martingale representation $\tilde{M}_{t}-\tilde{M}_{0}=\int_{0}^{t} \tilde{Z}_{s} d B_{s}$, and

$$
\tilde{Y}_{t}=\xi+\int_{t}^{T} f\left(s, Y_{s}, Z_{s}, H(K)_{s}^{b}\right) d s+\tilde{K}_{T}-\tilde{K}_{t}-\int_{t}^{T} \tilde{Z}_{s} d B_{s} .
$$

The remaining thing to check is whether Skorohod's equation holds for $(\tilde{Y}, \tilde{Z}, \tilde{K})$. Since $\tilde{Y}$ is the optional projection, and $\tilde{K}$ is the dual optional projection of $\hat{K}$, therefore,

$$
\begin{aligned}
\mathbb{E} \int_{0}^{T}\left(\tilde{Y}_{s}-S_{S}\right) d \tilde{K}_{s} & =\mathbb{E} \int_{0}^{T}\left(\tilde{Y}_{S}-S_{S}\right) d \hat{K}_{s} \\
& =\mathbb{E}\left(\int_{0}^{T}\left(\hat{Y}_{s}-S_{S}\right) d \hat{K}_{s}\right)^{o} .
\end{aligned}
$$

According to Skorohod's equation, $\tilde{K}$ increases only on $\left\{s: \hat{Y}_{s}-S_{s}=0\right\}$, so that $\int_{t}^{T}\left(\hat{Y}_{s}-S_{S}\right) d \hat{K}_{s}=0$ and, therefore, $\mathbb{E} \int_{t}^{T}\left(\tilde{Y}_{s}-S_{S}\right) d \tilde{K}_{s}=0$. While $\int_{0}^{T}\left(\tilde{Y}_{s}-\right.$ $\left.S_{S}\right) d \tilde{K}_{s} \geq 0$, which is a nonnegative random variable with expectation zero and, therefore, $\int_{0}^{T}\left(\tilde{Y}_{S}-S_{S}\right) d \tilde{K}_{s}=0$. It is clear from the definition and the Lipschitz condition (3.3) that

$$
(\tilde{Y}, \tilde{Z}, \tilde{K}) \in \mathcal{S}^{2}(0, T) \times \mathcal{H}_{d}^{2}(0, T) \times \mathcal{A}^{2}(0, T) .
$$

The mapping

$$
\mathfrak{L}: \mathcal{S}^{2}(0, T) \times \mathcal{H}_{d}^{2}(0, T) \times \mathcal{A}^{2}(0, T) \rightarrow \mathcal{S}^{2}(0, T) \times \mathcal{H}_{d}^{2}(0, T) \times \mathcal{A}^{2}(0, T)
$$

with $\mathfrak{L}(Y, Z, K)=(\tilde{Y}, \tilde{Z}, \tilde{K})$ is thus well-defined. Moreover, $\tilde{K} \in \mathcal{A}^{2}(0, T)$, which is an optional increasing process.

REMARK 3.1. This iteration procedure gives an "explicit" method to construct the solution of reflected BSDE. 
REMARK 3.2. If $(Y, Z, K)$ is a fixed point of $\mathfrak{L}$, then $(Y, Z, K)$ is a solution to the reflected BSDE (3.1)-(3.2). In fact, from Skorohod's equation, it is easy to check that $K^{o}=K$, therefore, $K$ is adapted, and $H(K)^{b}=H(K)$. Hence

$$
\begin{aligned}
Y_{t} & =\xi+\int_{t}^{T} f\left(s, Y_{s}, Z_{s}, H(K)_{s}\right) d s+\left(K_{T}^{o}-K_{t}^{o}\right)-\int_{t}^{T} Z_{s} d B_{s} \\
& =\xi+\int_{t}^{T} f\left(s, Y_{s}, Z_{s}, H(K)_{s}\right) d s+\left(K_{T}-K_{t}\right)-\int_{t}^{T} Z_{s} d B_{s}
\end{aligned}
$$

for all $t \in[0, T]$, which shows that $(Y, Z, K)$ is a solution.

3.2. Main estimates. In this part, we establish a priori estimates for $\mathfrak{L}(Y, Z$, $K)=(\tilde{Y}, \tilde{Z}, \tilde{K})$. We begin with an elementary fact:

LeMma 3.3. Let $\varphi, \psi$ be two continuous paths in $\mathbb{R}$. Then

$$
\left|\sup _{s \leq t} \varphi_{s}-\sup _{s \leq t} \psi_{s}\right| \leq \sup _{s \leq t}\left|\varphi_{s}-\psi_{s}\right| .
$$

The inequality is elementary, so we omit the proof here.

Suppose that

$$
(Y, Z, K),\left(Y^{\prime}, Z^{\prime}, K^{\prime}\right) \in \mathcal{S}^{2}(0, T) \times \mathcal{H}_{d}^{2}(0, T) \times \mathcal{A}^{2}(0, T)
$$

with $Y_{T}=Y_{T}^{\prime}=\xi$, and $Y \geq S, Y^{\prime} \geq S$.

Let

$$
(\tilde{Y}, \tilde{Z}, \tilde{K})=\mathfrak{L}(Y, Z, K) \quad \text { and } \quad\left(\tilde{Y}^{\prime}, \tilde{Z}^{\prime}, \tilde{K}^{\prime}\right)=\mathfrak{L}\left(Y^{\prime}, Z^{\prime}, K^{\prime}\right)
$$

Set $\alpha \geq 0$ to be chosen later, $D_{t}=e^{\alpha t}\left|Y_{t}-Y_{t}^{\prime}\right|^{2}$, and $\tilde{D}_{t}=e^{\alpha t}\left|\tilde{Y}_{t}-\tilde{Y}_{t}^{\prime}\right|^{2}$. If $X$ is a continuous process, then $\|X\|_{\alpha}=\sqrt{\mathbb{E} \int_{0}^{T} e^{\alpha t}\left|X_{t}\right|^{2} d t}$.

LEMmA 3.4. Suppose that $f$ satisfies the Lipschitz condition (3.3). Then, for $\alpha \geq 0, \varepsilon>0$ and $\varepsilon^{\prime}>0$,

$$
\begin{aligned}
\mathbb{E}\left[\tilde{D}_{0}\right] \leq & -\left(\alpha-\varepsilon C_{1}-\varepsilon^{\prime} C_{2}\right)\left\|\tilde{Y}-\tilde{Y}^{\prime}\right\|_{\alpha}^{2}-\left\|\tilde{Z}-\tilde{Z}^{\prime}\right\|_{\alpha}^{2} \\
& +\frac{2 C_{1}}{\varepsilon}\left(\left\|Y-Y^{\prime}\right\|_{\alpha}^{2}+\left\|Z-Z^{\prime}\right\|_{\alpha}^{2}\right) \\
& +\frac{2 C_{2}}{\varepsilon^{\prime}}\left\|H(K)^{b}-H\left(K^{\prime}\right)^{b}\right\|_{\alpha}^{2}
\end{aligned}
$$

where $C_{1}, C_{2}$ are the Lipschitz constants appearing in (3.3). 
PROOF. According to (3.10),

$$
\begin{aligned}
\tilde{Y}_{t}-\tilde{Y}_{t}^{\prime}= & \left(\tilde{M}_{t}-\tilde{M}_{t}^{\prime}\right)-\left(\tilde{K}_{t}-\tilde{K}_{t}^{\prime}\right) \\
& -\int_{0}^{t}\left(f\left(s, Y_{s}, Z_{s}, H(K)_{s}^{b}\right)-f\left(s, Y_{s}^{\prime}, Z_{s}^{\prime}, H\left(K^{\prime}\right)_{s}^{b}\right)\right) d s,
\end{aligned}
$$

where $\tilde{M}$ (resp., $\tilde{M}^{\prime}$ ) is the martingale part of $\tilde{Y}$ (resp., $\tilde{Y}^{\prime}$ ), given by (3.11). By Itô's formula,

$$
\begin{aligned}
\tilde{D}_{t}= & -\int_{t}^{T} e^{\alpha s} d\left(\tilde{Y}_{s}-\tilde{Y}_{s}^{\prime}\right)^{2}-\alpha \int_{t}^{T} e^{\alpha s}\left(\tilde{Y}_{s}-\tilde{Y}_{s}^{\prime}\right)^{2} d s \\
= & -\int_{t}^{T} e^{\alpha s} d\left\langle\tilde{M}-\tilde{M}^{\prime}\right\rangle_{s}-\alpha \int_{t}^{T} e^{\alpha s}\left(\tilde{Y}_{s}-\tilde{Y}_{s}^{\prime}\right)^{2} d s \\
& -\int_{t}^{T} 2 e^{\alpha s}\left(\tilde{Y}_{s}-\tilde{Y}_{s}^{\prime}\right) d\left(\tilde{Y}_{s}-\tilde{Y}_{s}^{\prime}\right) \\
= & -\alpha \int_{t}^{T} \tilde{D}_{s} d s-\int_{t}^{T} e^{\alpha s} d\left\langle\tilde{M}-\tilde{M}^{\prime}\right\rangle_{s}-2 \int_{t}^{T} e^{\alpha s}\left(\tilde{Y}_{s}-\tilde{Y}_{s}^{\prime}\right) d\left(\tilde{M}_{s}-\tilde{M}_{s}^{\prime}\right) \\
& +2 \int_{t}^{T} e^{\alpha s}\left(\tilde{Y}_{s}-\tilde{Y}_{s}^{\prime}\right) d\left(\tilde{K}_{s}-\tilde{K}_{s}^{\prime}\right) \\
& +2 \int_{t}^{T} e^{\alpha s}\left(\tilde{Y}_{s}-\tilde{Y}_{s}^{\prime}\right)\left(f\left(s, Y_{s}, Z_{s}, H(K)_{s}^{b}\right)-f\left(s, Y_{s}^{\prime}, Z_{s}^{\prime}, H\left(K^{\prime}\right)_{s}^{b}\right)\right) d s .
\end{aligned}
$$

After taking expectation, we obtain

$$
\begin{aligned}
\mathbb{E}\left[\tilde{D}_{t}\right]= & -\alpha \int_{t}^{T} \mathbb{E}\left(\tilde{D}_{s}\right) d s-\mathbb{E} \int_{t}^{T} e^{\alpha s} d\left\langle\tilde{M}-\tilde{M}^{\prime}\right\rangle_{s} \\
& +2 \mathbb{E} \int_{t}^{T} e^{\alpha s}\left(\tilde{Y}_{s}-\tilde{Y}_{s}^{\prime}\right) d\left(\tilde{K}_{s}-\tilde{K}_{s}^{\prime}\right) \\
& +2 \int_{t}^{T} \mathbb{E}\left\{e ^ { \alpha s } ( \tilde { Y } _ { s } - \tilde { Y } _ { s } ^ { \prime } ) \left[f\left(s, Y_{s}, Z_{s}, H(K)_{s}^{b}\right)\right.\right. \\
& \left.\left.-f\left(s, Y_{s}^{\prime}, Z_{s}^{\prime}, H\left(K^{\prime}\right)_{s}^{b}\right)\right]\right\} d s .
\end{aligned}
$$

Now we use an important observation due to [3] that

$$
\begin{aligned}
\mathbb{E} \int_{t}^{T} & e^{\alpha s}\left(\tilde{Y}_{s}-\tilde{Y}_{s}^{\prime}\right) d\left(\tilde{K}_{s}-\tilde{K}_{s}^{\prime}\right) \\
= & \mathbb{E} \int_{t}^{T} e^{\alpha s}\left(\tilde{Y}_{s}-S_{s}\right) d \tilde{K}_{s}+\mathbb{E} \int_{t}^{T} e^{\alpha s}\left(\tilde{Y}_{s}^{\prime}-S_{S}\right) d \tilde{K}_{s}^{\prime} \\
& \quad-\mathbb{E} \int_{t}^{T} e^{\alpha s}\left(\tilde{Y}_{s}-S_{s}\right) d \tilde{K}_{s}^{\prime}-\mathbb{E} \int_{t}^{T} e^{\alpha s}\left(\tilde{Y}_{s}^{\prime}-S_{s}\right) d \tilde{K}_{s}
\end{aligned}
$$

$\leq 0$ 
Putting this estimate into (3.15), we may deduce that

$$
\begin{aligned}
\mathbb{E}\left[\tilde{D}_{t}\right] \leq & -\alpha \int_{t}^{T} \mathbb{E}\left(\tilde{D}_{s}\right) d s-\mathbb{E} \int_{t}^{T} e^{\alpha s} d\left\langle\tilde{M}-\tilde{M}^{\prime}\right\rangle_{s} \\
& +2 \int_{t}^{T} e^{\alpha s} \mathbb{E}\left\{( \tilde { Y } _ { s } - \tilde { Y } _ { s } ^ { \prime } ) \left[f\left(s, Y_{s}, Z_{s}, H(K)_{s}^{b}\right)\right.\right. \\
& \left.\left.-f\left(s, Y_{s}^{\prime}, Z_{s}^{\prime}, H\left(K^{\prime}\right)_{s}^{b}\right)\right]\right\} d s .
\end{aligned}
$$

The standard method may be applied to handle the last integral on the right-hand side of (3.16) because $f$ is globally Lipschitz. In fact,

$$
\begin{aligned}
\mathbb{E}\left[\tilde{D}_{t}\right] \leq & -\alpha \int_{t}^{T} \mathbb{E}\left(\tilde{D}_{s}\right) d s-\mathbb{E} \int_{t}^{T} e^{\alpha s}\left|\tilde{Z}_{s}-\tilde{Z}_{s}\right|^{2} d s \\
& +2 C_{1} \int_{t}^{T} e^{\alpha s} \mathbb{E}\left(\left|\tilde{Y}_{s}-\tilde{Y}_{s}^{\prime}\right|\left(\left|Y_{s}-Y_{s}^{\prime}\right|+\left|Z_{s}-Z_{s}^{\prime}\right|\right)\right) d s \\
& +2 C_{2} \int_{t}^{T} e^{\alpha s} \mathbb{E}\left(\left|\tilde{Y}_{s}-\tilde{Y}_{s}^{\prime}\right|\left|H(K)_{s}^{b}-H\left(K^{\prime}\right)_{s}^{\mathrm{b}}\right|\right) d s \\
\leq & -\left(\alpha-\varepsilon C_{1}-\varepsilon^{\prime} C_{2}\right) \int_{t}^{T} \mathbb{E}\left(\tilde{D}_{s}\right) d s-\mathbb{E} \int_{t}^{T} e^{\alpha s}\left|\tilde{Z}_{s}-\tilde{Z}_{s}\right|^{2} d s \\
& +\frac{2 C_{1}}{\varepsilon} \mathbb{E} \int_{t}^{T} e^{\alpha s}\left(\left|Y_{s}-Y_{s}^{\prime}\right|^{2}+\left|Z_{s}-Z_{s}^{\prime}\right|^{2}\right) d s \\
& +\frac{2 C_{2}}{\varepsilon^{\prime}} \mathbb{E} \int_{t}^{T} e^{\alpha s}\left|H(K)_{s}^{b}-H\left(K^{\prime}\right)_{s}^{b}\right|^{2} d s
\end{aligned}
$$

which yields the required estimate.

The next estimate is also essential for the existence.

Proposition 3.5. Let $\left\|K-K^{\prime}\right\|_{\infty}^{2}=\mathbb{E}\left[\sup _{0 \leq t \leq T}\left|K_{s}-K_{s}^{\prime}\right|^{2}\right]$. Then

$$
\begin{aligned}
\left\|\tilde{K}-\tilde{K}^{\prime}\right\|_{\infty}^{2} \leq & \left(24 T C_{1}^{2}+4 C_{4}\right)\left(\left\|Y-Y^{\prime}\right\|_{0}^{2}+\left\|Z-Z^{\prime}\right\|_{0}^{2}\right) \\
& +24 T^{2} C_{2}^{2}\left\|K-K^{\prime}\right\|_{\infty}^{2},
\end{aligned}
$$

where $C_{4}$ is the constant appearing in the Burkhölder inequality.

Proof. Since

$$
\begin{aligned}
\tilde{K}_{t}= & \max \left[0, \max _{0 \leq s \leq T}\left\{-\left(\xi+\int_{s}^{T} f\left(r, Y_{r}, Z_{r}, H(K)_{r}^{b}\right) d r\right.\right.\right. \\
& \left.\left.\left.-S_{s}-\int_{s}^{T} Z_{r} d B_{r}\right)\right\}\right]
\end{aligned}
$$




$$
\begin{aligned}
& -\max \left[0, \max _{t \leq s \leq T}\left\{-\left(\xi+\int_{s}^{T} f\left(r, Y_{r}, Z_{r}, H(K)_{r}^{b}\right) d r\right.\right.\right. \\
& \left.\left.\left.-S_{S}-\int_{s}^{T} Z_{r} d B_{r}\right)\right\}\right]
\end{aligned}
$$

from which it follows that

$$
\begin{aligned}
\left|\tilde{K}_{t}-\tilde{K}_{t}^{\prime}\right|^{2} \leq & 4 T \int_{0}^{T}\left|f\left(s, Y_{s}, Z_{s}, H(K)_{s}^{b}\right)-f\left(s, Y_{s}^{\prime}, Z_{s}^{\prime}, H\left(K^{\prime}\right)_{s}^{b}\right)\right|^{2} d s \\
& +4\left|\sup _{0 \leq s \leq T} \int_{s}^{T}\left(Z_{r}-Z_{r}^{\prime}\right) d B_{r}\right|^{2} \\
\leq & 12 T C_{1}^{2} \int_{0}^{T}\left(\left|Y_{s}-Y_{s}^{\prime}\right|^{2}+\left|Z_{s}-Z_{s}^{\prime}\right|^{2}\right) d s \\
& +12 T C_{2}^{2} \int_{0}^{T}\left|\mathbb{E}\left[H(K)_{s}-H\left(K^{\prime}\right)_{s} \mid \mathcal{F}_{s}\right]\right|^{2} d s \\
& +4\left|\sup _{0 \leq s \leq T} \int_{s}^{T}\left(Z_{r}-Z_{r}^{\prime}\right) d B_{r}\right|^{2}
\end{aligned}
$$

Taking expectation and using (3.5), we obtain

$$
\begin{aligned}
\mathbb{E}\left|\tilde{K}_{t}-\tilde{K}_{t}^{\prime}\right|^{2} \leq & \left(12 T C_{1}^{2}+4 C_{4}\right) \mathbb{E} \int_{0}^{T}\left(\left|Y_{s}-Y_{s}^{\prime}\right|^{2}+\left|Z_{s}-Z_{s}^{\prime}\right|^{2}\right) d s \\
& +12 T C_{2}^{2} C_{3} \int_{0}^{T} \mathbb{E} \sup _{r}\left|K_{r}-K^{\prime}{ }_{r}\right|^{2} d s \\
\leq & \left(12 T C_{1}^{2}+4 C_{4}\right) \mathbb{E} \int_{0}^{T}\left(\left|Y_{s}-Y_{s}^{\prime}\right|^{2}+\left|Z_{s}-Z_{s}^{\prime}\right|^{2}\right) d s \\
& +12 T^{2} C_{2}^{2} C_{3}\left\|K-K^{\prime}\right\|_{\infty}^{2}
\end{aligned}
$$

which implies (3.18).

3.3. Existence and uniqueness theorem. We are now in a position to show the existence of a solution to (3.1)-(3.2).

THEOREM 3.6. There is a constant $C_{0}>0$ depending only on $C_{1}$ such that, if $T>0$ satisfies the condition that $C_{2} C_{3} T \leq C_{0}$, then there is a unique solution $(Y, Z, K)$ to the problem (3.1)-(3.2). Moreover, the reflected local time satisfies (3.6).

If $C_{2}=0$, that is, if the driver $f$ does not depend on $K$, then there is no restriction on $T . C_{1}, C_{2}$ and $C_{3}$ are the Lipschitz constants appearing in (3.3) and in (3.5), respectively. 
Proof. Let $\alpha \geq 0$ and $\beta>0$ to be chosen later, and define

$$
\left\|(Y, Z, K)-\left(Y^{\prime}, Z^{\prime}, K^{\prime}\right)\right\|_{\alpha, \beta}^{2}=\left\|Y-Y^{\prime}\right\|_{\alpha}^{2}+\left\|Z-Z^{\prime}\right\|_{\alpha}^{2}+\beta\left\|K-K^{\prime}\right\|_{\infty}^{2} .
$$

Let $(\tilde{Y}, \tilde{Z}, \tilde{K})=\mathfrak{L}(Y, Z, K)$ and $\left(\tilde{Y}^{\prime}, \tilde{Z}^{\prime}, \tilde{K}^{\prime}\right)=\mathfrak{L}\left(Y^{\prime}, Z^{\prime}, K^{\prime}\right)$. Then from (3.5),

$$
\begin{aligned}
\left\|H(K)^{b}-H\left(K^{\prime}\right)^{b}\right\|_{\alpha}^{2} & \leq \mathbb{E} \int_{t}^{T} e^{\alpha s} \mathbb{E}\left[\left|H(K)_{s}-H\left(K^{\prime}\right)_{s}\right|^{2} \mid \mathcal{F}_{t}\right] d s \\
& \leq \frac{e^{\alpha T}-1}{\alpha} C_{3}\left\|K-K^{\prime}\right\|_{\infty}^{2}
\end{aligned}
$$

together with (3.13) (we choose $\alpha \geq 0, \varepsilon \geq 0$ and $\varepsilon^{\prime} \geq 0$ such that $\alpha-\varepsilon C_{1}-\varepsilon^{\prime} C_{2}=$ 1), it follows that

$$
\begin{aligned}
&\left\|\tilde{Y}-\tilde{Y}^{\prime}\right\|_{\alpha}^{2}+\left\|\tilde{Z}-\tilde{Z}^{\prime}\right\|_{\alpha}^{2}+\beta\left\|\tilde{K}-\tilde{K}^{\prime}\right\|_{\infty}^{2} \\
& \leq {\left[\left(12 T C_{1}^{2}+4 C_{4}\right) \beta+\frac{2 C_{1}}{\varepsilon}\right]\left(\left\|Y-Y^{\prime}\right\|_{\alpha}^{2}+\left\|Z-Z^{\prime}\right\|_{\alpha}^{2}\right) } \\
&+\left(\frac{12 C_{2}^{2} T^{2} C_{3}}{\beta}+\frac{2 C_{2} C_{3}}{\varepsilon^{\prime} \beta} \frac{e^{\alpha T}-1}{\alpha}\right) \beta\left\|K-K^{\prime}\right\|_{\infty}^{2} .
\end{aligned}
$$

Choose $\varepsilon=8 C_{1}, \varepsilon^{\prime}=1, \alpha=1+8 C_{1}^{2}+C_{2}$ and $\beta=\frac{1}{16\left(3 T C_{1}^{2}+C_{4}\right) C_{3}}$. We may choose $C_{0}>0$ such that, if $C_{2} C_{3} T \leq C_{0}$, then

$$
6 C_{2}^{2} T^{2}+C_{2} \frac{e^{\left(1+8 C_{1}^{2}+C_{2}\right) T}-1}{1+8 C_{1}^{2}+C_{2}} \leq \frac{1}{64\left(3 T C_{1}^{2}+C_{4}\right) C_{3}}
$$

and, therefore,

$$
\frac{12 C_{2}^{2} C_{3}}{\beta} T^{2}+\frac{2 C_{2} C_{3}}{\beta} \frac{e^{\alpha T}-1}{\alpha} \leq \frac{1}{2} .
$$

With these choices of $\alpha, \beta, \varepsilon$ and $\varepsilon^{\prime}$, it follows from (3.19) that

$$
\left\|(\tilde{Y}, \tilde{Z}, \tilde{K})-\left(\tilde{Y}^{\prime}, \tilde{Z}^{\prime}, \tilde{K}^{\prime}\right)\right\|_{\alpha, \beta} \leq \frac{1}{\sqrt{2}}\left\|(Y, Z, K)-\left(Y^{\prime}, Z^{\prime}, K^{\prime}\right)\right\|_{\alpha, \beta},
$$

so there is a fixed point $(Y, Z, K)$, which is clearly a solution according to Remark 3.2.

The uniqueness of the solution follows from the contraction principle. We however supply a proof of the uniqueness which also shows continuous dependence of solutions on the parameters.

THEOREM 3.7. Under the same assumptions in Theorem 3.6, suppose that $\left(Y^{i}, Z^{i}, K^{i}\right)(i=1,2)$ are solutions of reflected BSDE (3.1) with parameters 
$\left(\xi^{i}, f^{i}, S^{i}\right)$, respectively. Let

$$
\begin{array}{rlrl}
\Delta Y & =Y^{1}-Y^{2}, & \Delta Z=Z^{1}-Z^{2}, & \Delta K=K^{1}-K^{2}, \\
\Delta \xi=\xi^{1}-\xi^{2}, & \Delta f=f^{1}-f^{2}, & \Delta S=S^{1}-S^{2} .
\end{array}
$$

Then

$$
\begin{aligned}
\mathbb{E}\left(\sup _{0 \leq t \leq T}\left|\Delta Y_{t}\right|^{2}+\int_{0}^{T}\left|\Delta Z_{t}\right|^{2} d t+\sup _{0 \leq t \leq T}\left|\Delta K_{t}\right|^{2}\right) \\
\leq C \mathbb{E}\left(\Delta \xi^{2}+\int_{0}^{T}\left|\Delta f\left(t, Y_{t}^{1}, Z_{t}^{1}, H\left(K^{1}\right)_{t}\right)\right|^{2} d t\right) \\
\quad+C \Psi_{T}^{\frac{1}{2}}\left[\mathbb{E}\left(\sup _{0 \leq t \leq T}\left|\Delta S_{t}\right|\right)^{2}\right]^{\frac{1}{2}},
\end{aligned}
$$

where $C$ depends only on $C_{1}, C_{2}, C_{3}$ and $C_{2} C_{3} T$, and

$$
\begin{aligned}
\Psi_{T}= & \mathbb{E}\left(\left|\xi^{1}\right|^{2}+\left|\xi^{2}\right|^{2}\right)+\mathbb{E}\left[\left(\sup _{0 \leq t \leq T}\left(S_{t}^{1}\right)^{+}\right)^{2}+\left(\sup _{0 \leq t \leq T}\left(S_{t}^{2}\right)^{+}\right)^{2}\right] \\
& +\mathbb{E} \int_{0}^{T}\left(\left|f_{0}^{1}(t)\right|^{2}+\left|f_{0}^{2}(t)\right|^{2}\right) d t,
\end{aligned}
$$

where $f_{0}^{i}(t)=f^{i}(t, 0,0,0), i=1,2$.

PROOF. Applying Itô's formula to $\left|\Delta Y_{t}\right|^{2}$, then taking expectation together with the fact that

$$
\int_{t}^{T}\left(\Delta Y_{s}-\Delta S_{S}\right) d\left(\Delta K_{S}\right) \leq 0
$$

we get

$$
\begin{aligned}
\mathbb{E}\left(\left|\Delta Y_{t}\right|^{2}+\int_{t}^{T}\left|\Delta Z_{s}\right|^{2} d s\right) \\
=\mathbb{E}\left(\Delta \xi^{2}+2 \int_{t}^{T} \Delta Y_{s} \Delta f\left(s, Y_{s}^{1}, Z_{s}^{1}, H\left(K^{1}\right)_{s}\right) d s\right) \\
\quad+2 \mathbb{E}\left(\int_{t}^{T} \Delta Y_{s}\left(f^{2}\left(s, Y_{s}^{1}, Z_{s}^{1}, H\left(K^{1}\right)_{s}\right)-f^{2}\left(s, Y_{s}^{2}, Z_{s}^{2}, H\left(K^{2}\right)_{s}\right)\right) d s\right) \\
\quad+2 \mathbb{E} \int_{t}^{T} \Delta S_{s} d\left(\Delta K_{s}\right) .
\end{aligned}
$$


Together with (3.3), we then deduce that

$$
\begin{aligned}
\mathbb{E}\left|\Delta Y_{t}\right|^{2} \leq & \mathbb{E}\left(\Delta \xi^{2}+\int_{t}^{T}\left|\Delta f\left(s, Y_{s}^{1}, Z_{s}^{1}, H\left(K^{1}\right)_{s}\right)\right|^{2} d s\right) \\
& +\left(2+2 C_{1}+C_{1}^{2}\right) \mathbb{E} \int_{t}^{T}\left|\Delta Y_{s}\right|^{2} d s+C_{2}^{2} C_{3} T^{2} \mathbb{E}\left[\sup _{0 \leq t \leq T}\left|\Delta K_{t}\right|^{2}\right] \\
& +\mathbb{E}\left[\sup _{0 \leq t \leq T}\left|\Delta S_{t}\right|\left(K_{T}^{1}+K_{T}^{2}\right)\right],
\end{aligned}
$$

which yields estimates for $\mathbb{E} \int_{t}^{T}\left|\Delta Y_{S}\right|^{2} d s$ and $\int_{0}^{T}\left|\Delta Z_{S}\right|^{2} d s$ via Gronwall's inequality. By (3.6),

$$
\begin{aligned}
K_{t}^{i}= & \max \left[0, \max _{0 \leq s \leq T}\left\{-\left(\xi^{i}+\int_{s}^{T} f^{i}\left(r, Y_{r}^{i}, Z_{r}^{i}, H\left(K^{i}\right)_{r}^{b}\right) d r\right.\right.\right. \\
& \left.\left.\left.-S_{s}^{i}-\int_{s}^{T} Z_{r}^{i} d B_{r}\right)\right\}\right] \\
& -\max \left[0, \max _{t \leq s \leq T}\left\{-\left(\xi^{i}+\int_{s}^{T} f^{i}\left(r, Y_{r}^{i}, Z_{r}^{i}, H\left(K^{i}\right)_{r}^{b}\right) d r\right.\right.\right. \\
& \left.\left.\left.-S_{s}^{i}-\int_{s}^{T} Z_{r}^{i} d B_{r}\right)\right\}\right]
\end{aligned}
$$

for $i=1,2$, and using similar arguments as in the proof of Proposition 3.5, we obtain

$$
\begin{aligned}
\mathbb{E}\left[\sup _{0 \leq t \leq T}\left|\Delta K_{t}\right|^{2}\right] \leq & 4 \mathbb{E}\left(\Delta \xi^{2}+\int_{t}^{T}\left|\Delta f\left(s, Y_{s}^{1}, Z_{s}^{1}, H\left(K^{1}\right)_{s}\right)\right|^{2} d s\right. \\
& \left.+\mathbb{E} \sup _{0 \leq t \leq T}\left|\Delta S_{t}\right|^{2}\right) \\
& +\left(12 T C_{1}^{2}+4 C_{4}\right) \mathbb{E}\left[\int_{0}^{T}\left(\left|\Delta Y_{s}\right|^{2}+\left|\Delta Z_{s}\right|^{2}\right) d s\right] \\
& +12 T^{2} C_{2}^{2} C_{3} \mathbb{E}\left[\sup _{0 \leq t \leq T}\left|\Delta K_{t}\right|^{2}\right] .
\end{aligned}
$$

Putting these estimations together with similar technique in proving Theorem 3.6, we can choose $C_{0}>0$ small enough such that, if $C_{2} C_{3} T \leq C_{0}$, so that (3.22) holds.

\section{Application in finance.}

4.1. Optimal stopping problem. In [3], it is proven that the value processes of the optimal stopping problem can be presented as solutions of reflected BSDEs. In 
financial market, solutions of classic BSDEs can be considered as recursive utility of an investor, which means that the wealth of the investor will affect his decision, that is, the utility function. This model can also be applied to the pricing problem under non-Black-Scholes framework; cf. [4] for more details. With the help of the relationship between solutions of reflected BSDEs and value processes of some optimal stopping problems, it is known that the reflected BSDE is useful in the study of mixed optimal control problems with recursive utility, to price Americantype options under non-Black-Scholes framework. Solutions of reflected BSDEs with resistance (3.1), can be considered as value processes of optimal stopping problems, according to the arguments as for Proposition 2.3 in [3]. Namely, we have the following.

PROPOSITION 4.1. If $(Y, Z, K)$ is the solution of reflected BSDE with resistance (3.1) and (3.2), then for each $t \in[0, T]$

$$
Y_{t}=\operatorname{ess} \sup _{\tau \in \mathcal{T}_{t}} \mathbb{E}\left[\int_{t}^{\tau} f\left(s, Y_{s}, Z_{s}, H(K)_{s}\right) d s+S_{\tau} 1_{\{\tau<T\}}+\xi 1_{\{\tau=T\}} \mid \mathcal{F}_{t}\right],
$$

where $\mathcal{T}_{t}$ is the set of all stopping times valued in $[t, T]$.

However, this optimal stopping problem is not standard, the method of Snell envelopes cannot be utilized directly to obtain the solution, since the value process also depends on the increasing process $K$.

4.2. Super-hedging problem with wealth constraint. Following [4], in this part, we consider another application of reflected BSDEs introduced in the previous section in finance. Consider an investor whose actions cannot affect market prices. The investor can decide to put at time $t \in[0, T]$ an amount $\pi_{t}=\left(\pi_{t}^{i}\right)_{0 \leq i \leq n}$ of his wealth $V_{t}$ in risky assets, and to allocate his consumption $C_{t}$. His decision can only be based on the current information $\left(\mathcal{F}_{t}\right)$, that is, the processes $\pi_{t}$ and $C_{t}$ are predictable. Here, $C$ is an increasing process with $C_{0}=0$. In practice, $C_{t}=\int_{0}^{t} c_{s} d s$, which is cumulative amount of consumption from 0 to $t$, though we do not assume this. It can be interpreted as liquidity necessary under some constraint. We assume that the triple $(V, \pi, C)$ satisfies following stochastic differential equation:

$$
d V_{t}=b\left(t, V_{t}, \pi_{t}, H(C)_{t}\right) d t-d C_{t}+\sigma_{t} \pi_{t} d B_{t} .
$$

In the classical financial model, we have $b(s, y, z)=-\left(r_{s} y+\sigma_{s}^{-1}\left(\mu_{s}-r_{s}\right) z\right)$, where $r$ is the interest of bank, $\mu$ is the expected return of risky asset, $\sigma$ is the volatility.

Let us consider the case that the consumption also has impact on the wealth through the market mechanics described by mapping

$$
b:[0, T] \times \Omega \times \mathbb{R} \times \mathbb{R}^{d} \times \mathbb{R}^{+} \rightarrow \mathbb{R},
$$


where $H$ is a mapping defined on the space of consumption processes, which may depend on the whole history of $C$. We introduce the following definition of superstrategy (cf. [5] and [4]).

DEFinition 4.2. A super-strategy is a triple process $(V, \pi, C)$, where $V$ is the market value (or wealth process), $\pi$ is the portfolio process, and $C$ is the consumption process, such that

$$
\begin{aligned}
d V_{t} & =b\left(t, V_{t}, \pi_{t}, H(C)_{t}\right) d t-d C_{t}+\sigma_{t} \pi_{t} d B_{t}, \\
\int_{0}^{T}\left|\pi_{t}\right|^{2} d t & <+\infty, \quad \mathbb{P} \text {-a.s., }
\end{aligned}
$$

and $C$ is an increasing, right-continuous, adapted process with $C_{0}=0$.

Let $\xi$ be a positive contingent claim, with the settled time $T$ which is an $\mathcal{F}_{T}$ measurable random variable. It can be regarded as a financial target of an investor, or a contract which pays $\xi$ at maturity $T$.

DEFINITION 4.3. (i) A super-hedging strategy against $\xi$ is a self-financing strategy $(V, \pi, C)$ such that $V_{T}=\xi$. Let $\mathcal{H}(\xi)$ denote the class of super-hedging strategies against $\xi$. If $\mathcal{H}(\xi)$ is nonempty, $\xi$ is called super-hedgeable.

(ii) The upper price $V_{0}$ (or upper initial invest $V_{0}$ ) at time 0 of the superhedgeable claim $\xi$ is the smallest initial endowment needed to hedge $\xi$, that is,

$$
V_{0}=\inf \left\{x \geq 0 ; \exists(V, \pi, C) \in \mathcal{H}(\xi) \text { such that } V_{0} \text { that is, }=x\right\} .
$$

The corresponding self-financing strategy $(V, \pi, C)$, if it exists, is called the smallest super-hedging strategy.

The definitions of super-hedging and super-strategy are often used in the situation where there is constraint or il-liquidity in the market. The upper price $V_{0}$ of $\xi$ in such market is the smallest initial capital which the investor should prepared to super-hedge $\xi$. On the other hand, if an investor has an initial capital $x$ smaller than $V_{0}$, then he cannot super-hedge $\xi$ by super-hedge strategy. In the paper [5], the upper price is called the selling price.

We are interested in the following.

PROBlem 4.4. Suppose that the market has a bankrupt constraint, that is, the wealth is forced to stay above a given level $S_{t}$, that is, $V_{t} \geq S_{t}, t \in[0, T]$, $S$ is a given random process satisfying suitable integrability condition. Given a contingent claim $\xi$, under (4.2), what is the lower price for it? How to find its smallest super-hedging strategy? 
Obviously under bankrupt constraint the smallest super-hedging strategy must satisfy the integrability condition, that is,

$$
\int_{0}^{T}\left(V_{t}-S_{t}\right) d C_{t}=0
$$

Applying results of reflected BSDE with nonlinear resistance, we have immediately the following.

PROPOSITION 4.5. If assumptions in Theorem 3.6 are satisfied for $b$ and $H$, then $\xi \in \mathcal{L}^{2}\left(\mathcal{F}_{T}\right)$ is super-hedgeable. The upper price $Y_{0}$ of contingent claim $\xi$ is the solution of the following reflected BSDE:

$$
\begin{aligned}
& Y_{t}=\xi-\int_{t}^{T} b\left(s, Y_{s}, Z_{s}, H(K)_{s}\right) d s+K_{T}-K_{t}-\int_{t}^{T} Z_{s} d B_{s}, \\
& Y_{t} \geq S_{t} \quad \text { for } t \leq T \quad \text { and } \quad \int_{0}^{T}\left(Y_{t}-S_{t}\right) d K_{t}=0 .
\end{aligned}
$$

In this case, the solution $\left(Y, \sigma^{-1} Z, K\right)=(V, \pi, C)$ is the smallest superhedging strategy.

5. Reflected BSDE with the density process. In the previous sections, we have discussed the following reflected BSDE with nonlinear resistance:

$$
\begin{aligned}
& Y_{t}=\xi+\int_{t}^{T} f\left(s, Y_{s}, Z_{s}, H(K)_{s}\right) d s+K_{T}-K_{t}-\int_{t}^{T} Z_{s} d B_{s}, \\
& Y_{t} \geq S_{t} \quad \text { for } t \leq T \quad \text { and } \quad \int_{0}^{T}\left(Y_{t}-S_{t}\right) d K_{t}=0 .
\end{aligned}
$$

We know from Corollary 2.2 that if $S$ is an Itô process

$$
S_{t}=S_{0}+\int_{0}^{t} u_{s} d s+\int_{0}^{t} \sigma_{s} d B_{s}
$$

then $K$ is absolutely continuous with respect to Lebesgue measure, that is, there exists a density process $k$ such that $K_{t}=\int_{0}^{t} k_{s} d s$. Furthermore, we have from Proposition 2.1 and Corollary 2.2 that

$$
1_{\left\{Y_{t}=S_{t}\right\}}\left(Z_{t}-\sigma_{t}\right)=0
$$

and

$$
\begin{aligned}
K_{t}+L_{t}^{Y} & =-\int_{0}^{t} 1_{\left\{Y_{s}=S_{s}\right\}}\left(f\left(s, Y_{s}, Z_{s}, H(K)_{s}\right)+u_{s}\right) d s \\
& =-\int_{0}^{t} 1_{\left\{Y_{s}=S_{s}\right\}}\left(f\left(s, S_{s}, \sigma_{s}, H(K)_{s}\right)+u_{s}\right) d s .
\end{aligned}
$$


Moreover, there exists a process $\left(\alpha_{t}\right)$ valued in $[0,1]$, such that, for $s \in[0, T]$,

$$
k_{s}=\alpha_{s} 1_{\left\{Y_{s}=S_{s}\right\}}\left(f\left(s, S_{s}, \sigma_{s}, H(K)_{s}\right)+u_{s}\right)^{-} .
$$

An interesting case to consider is the RBSDE with $H(K)_{s}=k_{s}$. It is easy to check that for this choice of $H$, inequality (3.5) is not satisfied. Therefore, we have to derive different estimates in order to solve the following problem:

$$
\begin{aligned}
& Y_{t}=\xi+\int_{t}^{T} f\left(s, Y_{s}, Z_{s}, k_{s}\right) d s+K_{T}-K_{t}-\int_{t}^{T} Z_{s} d B_{s}, \\
& Y_{t} \geq S_{t} \quad \text { for } t \leq T \quad \text { and } \quad \int_{0}^{T}\left(Y_{t}-S_{t}\right) d K_{t}=0 .
\end{aligned}
$$

Equivalently,

$$
\begin{aligned}
& Y_{t}=\xi+\int_{t}^{T}\left(f\left(s, Y_{s}, Z_{s}, k_{s}\right)+k_{s}\right) d s-\int_{t}^{T} Z_{s} d B_{s} \\
& Y_{t} \geq S_{t} \quad \text { for } t \leq T, k_{t} \geq 0 \quad \text { and } \quad \int_{0}^{T}\left(Y_{t}-S_{t}\right) k_{t} d t=0 .
\end{aligned}
$$

THEOREM 5.1. Under assumption (3.3), and $\bar{f}(k)=f\left(t, S_{t}, \sigma_{t}, k\right)$ for $k \in \mathbb{R}^{+}$. If (i) $\bar{f}$ is increasing in $k$, or (ii) $\left|\bar{f}(k)-\bar{f}\left(k^{\prime}\right)\right| \leq C_{k}\left|k^{\prime}-k\right|$, for $k, k^{\prime}>0$, with $C_{k}<1$, then for any $\xi \in \mathcal{L}^{2}\left(\mathcal{F}_{T}\right)$, there exists at least one triple $\left(Y_{t}, Z_{t}, k_{t}\right)$ which is the solution of reflected BSDE (5.4).

PROOF. We will construct the solution directly via auxiliary BSDEs, not through the Picard iteration. Thanks to (5.1) and (5.3), the problem (5.4) may be written as

$$
\begin{aligned}
Y_{t}= & \xi+\int_{t}^{T}\left(f\left(s, Y_{s}, Z_{s}, k_{s}\right)+k_{s}\right) d s-\int_{t}^{T} Z_{s} d B_{s} \\
= & \xi+\int_{t}^{T} f\left(s, Y_{s}, Z_{s}, 0\right) d s-\int_{t}^{T} Z_{s} d B_{s} \\
& +\int_{t}^{T} 1_{\left\{Y_{s}=S_{s}\right\}}\left(f\left(s, Y_{s}, Z_{s}, k_{s}\right)-f\left(s, Y_{s}, Z_{s}, 0\right)+k_{s}\right) d s \\
= & \xi-\int_{t}^{T} Z_{s} d B_{s}+\int_{t}^{T} f\left(s, Y_{s}, Z_{s}, 0\right) d s \\
& +\int_{t}^{T} 1_{\left\{Y_{s}=S_{s}\right\}}\left(f\left(s, S_{s}, \sigma_{s}, k_{s}\right)-f\left(s, S_{s}, \sigma_{s}, 0\right)+k_{s}\right) d s .
\end{aligned}
$$

We therefore consider the reflected BSDE without resistance,

$$
\begin{aligned}
& \tilde{Y}_{t}=\xi+\int_{t}^{T} f\left(s, \tilde{Y}_{s}, \tilde{Z}_{s}, 0\right) d s+\tilde{K}_{T}-\tilde{K}_{t}-\int_{t}^{T} \tilde{Z}_{s} d B_{s}, \\
& \tilde{Y}_{t} \geq S_{t} \quad \text { for } t \leq T \quad \text { and } \quad \int_{0}^{T}\left(\tilde{Y}_{t}-S_{t}\right) d \tilde{K}_{t}=0 .
\end{aligned}
$$


Since $f$ satisfies Lipschitz condition (3.3), there exists a triple $(\tilde{Y}, \tilde{Z}, \tilde{K})$ which solves (5.6). Since $S$ is an Itô process, $\tilde{K}$ is absolutely continuous with respect to Lebesgue measure, thus there exists an adapted process $\tilde{k}_{s}$ such that $\tilde{K}_{t}=\int_{0}^{t} \tilde{k}_{s} d s$.

We want to find a solution $k_{s}$ to the following functional equation:

$$
f\left(s, S_{s}, \sigma_{s}, k_{s}\right)-f\left(s, S_{s}, \sigma_{s}, 0\right)+k_{s}=\tilde{k}_{s} .
$$

In fact, in the case that $\bar{f}$ is increasing in $k$, then define $\tilde{f}(k)=\bar{f}(k)-\bar{f}(0)+$ $k$, which is still increasing in $k$. Notice that $\tilde{f}(0)=0$. Thus $(5.7)$ has a positive solution

$$
k_{s}=\tilde{f}^{-1}\left(\tilde{k}_{s}\right) \geq 0 .
$$

If (ii) holds, so that $\bar{f}$ is Lipschitz with Lipschitz constant smaller than 1. Define $\tilde{f}(k)=\bar{f}(k)-\bar{f}(0)+k$. Then for $k \leq k^{\prime}$, we have

$$
\begin{aligned}
\tilde{f}\left(k^{\prime}\right)-\tilde{f}(k) & =\bar{f}\left(k^{\prime}\right)-\bar{f}(k)+k^{\prime}-k \\
& \geq-C_{k}\left|k^{\prime}-k\right|+k^{\prime}-k \\
& =\left(1-C_{k}\right)\left(k^{\prime}-k\right) \geq 0 .
\end{aligned}
$$

This implies that $\tilde{f}(k)$ is also increasing in $k$. Since $\tilde{f}(0)=0$, equation (5.7) admits a positive solution, $\tilde{k}_{s} \geq 0$, thus

$$
k_{s}=\tilde{f}^{-1}\left(\tilde{k}_{s}\right) \geq 0 .
$$

Since $\tilde{f}(0)=0$ at $(t, \omega), \tilde{k}_{t}=0$ implies that $k_{t}=0$, and since $\int_{0}^{T}\left(\tilde{Y}_{t}-\right.$ $\left.S_{t}\right) \tilde{k}_{t} d t=0$, we know that $\int_{0}^{T}\left(\tilde{Y}_{t}-S_{t}\right) k_{t} d t=0$. Let $Y=\tilde{Y}, Z=\tilde{Z}$. Then the triple $\left(Y_{t}, Z_{t}, k_{t}\right)_{0 \leq t \leq T}$ is a solution to (5.4).

REMARK 5.2. The key step in solving equation (5.4) is to find a solution in (5.7). Similar to (ii), we give another sufficient assumption for $f: f$ is decreasing in $k$ and $\left|\bar{f}(k)-\bar{f}\left(k^{\prime}\right)\right| \leq C_{k}\left|k^{\prime}-k\right|$, for $k, k^{\prime}>0$, with $C_{k}>1$.

REMARK 5.3. If $f$ differentiable in $k$, for $k \geq 0$, then a sufficient condition for (i) or (ii) in the previous theorem to hold is that $\frac{d f}{d k}>-1$, for $k \geq 0$. And for previous remark, it should be $\frac{d f}{d k}<-1$, for $k \geq 0$.

However the uniqueness is not obvious, if we change process $\mathbf{0}$ to another positive square integrable process $\bar{k}_{t}$ in 5.5 , the procedure will also give us a solution $\left(\hat{Y}_{t}, \hat{Z}_{t}, \hat{k}_{t}\right)$, under the same assumption, which may not coincide with the solution $\left(Y_{t}, Z_{t}, k_{t}\right)$ we get from process 0 , as they are constructed from different reflected BSDEs.

Nevertheless, we can still prove a uniqueness result under a stronger assumption than those in Theorem 5.1, but similar to the one in Remark 5.3. 
PROPOSITION 5.4. If $f$ is differential in $k$ with $\frac{d f}{d k}>-1$ for any $(t, \omega, y, z)$ and $k \geq 0$, then the reflected BSDE (5.4) admits at most one unique solution.

PROOF. The idea of the proof is similar to classical techniques for uniqueness of BSDE. Assume that there are two triples $\left(Y^{1}, Z^{1}, k^{1}\right)$ and $\left(Y^{2}, Z^{2}, k^{2}\right)$ which satisfy (5.4). We will consider the difference $\left|Y^{1}-Y^{2}\right|$ under suitable norm as usual. Applying Itô formulae to $\left|Y^{1}-Y^{2}\right|^{2}$ and taking expectation, we get

$$
\begin{aligned}
\mathbb{E} \mid Y_{t}^{1} & -\left.Y_{t}^{2}\right|^{2}+\mathbb{E} \int_{t}^{T}\left|Z_{s}^{1}-Z_{s}^{2}\right|^{2} d s \\
& =2 \mathbb{E} \int_{t}^{T}\left(Y_{s}^{1}-Y_{s}^{2}\right)\left(f\left(Y_{s}^{1}, Z_{s}^{1}, k_{s}^{1}\right)+k_{s}^{1}-f\left(Y_{s}^{2}, Z_{s}^{2}, k_{s}^{2}\right)-k_{s}^{2}\right) d s .
\end{aligned}
$$

Set $\hat{f}(t, y, z, k)=f(t, y, z, k)+k$. Obviously, it is increasing in $k$ in view of $\frac{d f}{d k}>$ -1 . Consider the reflected BSDEs with $\left(Y^{1}, Z^{1}, k^{1}\right)$ and $\left(Y^{2}, Z^{2}, k^{2}\right)$ as BSDEs with given process $k^{1}$ and $k^{2}$,

$$
\begin{aligned}
& Y_{t}^{1}=\xi+\int_{t}^{T} \hat{f}\left(s, Y_{s}^{1}, Z_{s}^{1}, k_{s}^{1}\right) d s-\int_{t}^{T} Z_{s}^{1} d B_{s}, \\
& Y_{t}^{2}=\xi+\int_{t}^{T} \hat{f}\left(s, Y_{s}^{2}, Z_{s}^{2}, k_{s}^{2}\right) d s-\int_{t}^{T} Z_{s}^{2} d B_{s} .
\end{aligned}
$$

Since if $k_{t}^{1} \geq k_{t}^{2}$, then $\hat{f}\left(t, y, z, k_{t}^{1}\right) \geq \hat{f}\left(t, y, z, k_{t}^{2}\right)$, by the comparison theorem, $Y_{t}^{1} \geq Y_{t}^{2}$. Similarly, $k_{t}^{1} \leq k_{t}^{2}$ implies that $Y_{t}^{1} \leq Y_{t}^{2}$. Thus we always have

$$
\left(k_{t}^{1}-k_{t}^{2}\right)\left(Y_{t}^{1}-Y_{t}^{2}\right) \geq 0 \text {. }
$$

On the other hand, two triples $\left(Y^{1}, Z^{1}, k^{1}\right)$ and $\left(Y^{2}, Z^{2}, k^{2}\right)$ satisfy the constraint that $\int_{0}^{T}\left(Y_{t}^{i}-S_{t}\right) k_{t}^{i} d t=0$, for $i=1,2$. By a simple calculation,

$$
\begin{aligned}
\int_{0}^{T}\left(Y_{t}^{1}-Y_{t}^{2}\right)\left(k_{t}^{1}-k_{t}^{2}\right) d t \\
=\int_{0}^{T}\left(Y_{t}^{1}-S_{t}\right) k_{t}^{1} d t+\int_{0}^{T}\left(Y_{t}^{2}-S_{t}\right) k_{t}^{2} d t-\int_{0}^{T}\left(Y_{t}^{1}-S_{t}\right) k_{t}^{2} d t \\
\quad-\int_{0}^{T}\left(Y_{t}^{2}-S_{t}\right) k_{t}^{1} d t \\
\quad \leq 0
\end{aligned}
$$

Hence $\left(k_{t}^{1}-k_{t}^{2}\right)\left(Y_{t}^{1}-Y_{t}^{2}\right)=0$ a.e. on $[0, T]$. By (5.8), with the help of (3.3), it is easy to get that

$$
\mathbb{E}\left|Y_{t}^{1}-Y_{t}^{2}\right|^{2} \leq\left(C_{1}+C_{1}^{2}\right) \mathbb{E} \int_{t}^{T}\left|Y_{s}^{1}-Y_{s}^{2}\right|^{2} d t
$$

By Gronwall's inequality, $Y_{t}^{1}=Y_{t}^{2}$, on $[0, T]$, and, therefore, $Z_{t}^{1}=Z_{t}^{2}$ and $k_{t}^{1}=k_{t}^{2}$. 
REMARK 5.5. This result is not in contradiction with the previous comments. In fact, we can only solve the equation by using process $\mathbf{0}$ in (5.5). If we replace process $\mathbf{0}$ by another positive process $\bar{k}_{s}$, then we cannot construct a triple to be the solution of (5.4) in similar way of solving equations (5.6) and (5.7). Since on $\left\{\tilde{k}_{s}=0\right\}$, by comparison theorem, $\bar{Y} \geq \tilde{Y} \geq S_{t}$, we would have

$$
f\left(s, S_{s}, \sigma_{s}, k_{s}\right)+k_{s}-f\left(s, S_{s}, \sigma_{s}, \bar{k}_{s}\right)=0 .
$$

Since $f(t, y, z, k)+k$ is increasing in $k, k_{s} \leq \bar{k}_{s}$, however, 0 may not be a solution of the above equation.

Acknowledgements. This research was carried out while the second author was visiting the Mathematical Institute, Oxford, and the Oxford-Man Institute.

\section{REFERENCES}

[1] BAnK, P. and El KAROUI, N. (2004). A stochastic representation theorem with applications to optimization and obstacle problems. Ann. Probab. 32 1030-1067. MR2044673

[2] Bismut, J.-M. (1976). Théorie probabiliste du contrôle des diffusions. Mem. Amer. Math. Soc. 4 xiii+130. MR0453161

[3] El Karoui, N., Kapoudjian, C., Pardoux, E., Peng, S. and Quenez, M. C. (1997). Reflected solutions of backward SDE's, and related obstacle problems for PDE's. Ann. Probab. 25 702-737. MR1434123

[4] El Karoui, N., Peng, S. and Quenez, M. C. (1997). Backward stochastic differential equations in finance. Math. Finance 7 1-71. MR1434407

[5] El Karoui, N. and QuenEZ, M.-C. (1995). Dynamic programming and pricing of contingent claims in an incomplete market. SIAM J. Control Optim. 33 29-66. MR1311659

[6] HamadÈne, S. (2002). Reflected BSDE's with discontinuous barrier and application. Stoch. Stoch. Rep. 74 571-596. MR1943580

[7] HE, S. W., WANG, J. G. and YAn, J. A. (1992). Semimartingale Theory and Stochastic Calculus. Kexue Chubanshe (Science Press), Beijing; CRC Press, Boca Raton, FL. MR1219534

[8] Karatzas, I. and Shreve, S. E. (1991). Brownian Motion and Stochastic Calculus, 2nd ed. Graduate Texts in Mathematics 113. Springer, New York. MR1121940

[9] Kobylanski, M., Lepeltier, J. P., Quenez, M. C. and Torres, S. (2002). Reflected BSDE with superlinear quadratic coefficient. Probab. Math. Statist. 22 51-83. MR1944142

[10] Lepeltier, J.-P., Matoussi, A. and XU, M. (2005). Reflected backward stochastic differential equations under monotonicity and general increasing growth conditions. Adv. in Appl. Probab. 37 134-159. MR2135157

[11] LePeltier, J.-P. and XU, M. (2005). Penalization method for reflected backward stochastic differential equations with one r.c.1.1. barrier. Statist. Probab. Lett. 75 58-66. MR2185610

[12] LEPELTIER, J.-P. and XU, M. (2007). Reflected BSDE with quadratic growth and unbounded terminal value. arXiv:0711.06191.

[13] MA, J. and WANG, Y. (2009). On variant reflected backward SDEs, with applications. J. Appl. Math. Stoch. Anal. Art. ID 854768, 26. MR2511615

[14] Matoussi, A. (1997). Reflected solutions of backward stochastic differential equations with continuous coefficient. Statist. Probab. Lett. 34 347-354. MR1467440

[15] Pardoux, É. and Peng, S. G. (1990). Adapted solution of a backward stochastic differential equation. Systems Control Lett. 14 55-61. MR1037747 
[16] Peng, S. and XU, M. (2005). The smallest $g$-supermartingale and reflected BSDE with single and double $L^{2}$ obstacles. Ann. Inst. Henri Poincaré Probab. Stat. 41 605-630. MR2139035

[17] Peng, S. G. (1991). Probabilistic interpretation for systems of quasilinear parabolic partial differential equations. Stoch. Stoch. Rep. 37 61-74. MR1149116

[18] XU, M. (2008). Backward stochastic differential equations with reflection and weak assumptions on the coefficients. Stochastic Process. Appl. 118 968-980. MR2418253

[19] Zhang, J. (2004). A numerical scheme for BSDEs. Ann. Appl. Probab. 14 459-488. MR2023027

Mathematical institute

UNIVERSITY OF OXFORD

ANDREW WILES BUILDING

RADCLIFFE OBSERVATORY QUARTER

WOODSTOCK ROAD

OXFORD OX2 6GG

ENGLAND

E-MAIL:qianz@maths.ox.ac.uk
INSTITUTE OF MATHEMATICS

RCSDS AND NCMIS

ACADEMY OF MATHEMATICS

AND SySTEMS SCIENCE

Chinese ACAdemy of Sciences

BEIJING 100190

CHINA

E-MAIL: xumy@amss.ac.cn 